\title{
Review: screening for depression reduces persistent depression
}

\author{
Pignone MP, Gaynes BN, Rushton JL, et al. Screening for depression in adults: a summary of the evidence for the U.S. \\ Preventive Services Task Force. Ann Intern Med 2002 May 21;136:765-76.

\section{QUESTION: Is screening for depression in adults effective in increasing diagnosis and treatment rates and improving clinical outcomes?}

\section{Data sources}

Studies were identified by searching Medline (19662001); the Cochrane database on depression, neurosis, and anxiety disorders; hand searching bibliographies of relevant studies; and contacting experts in the field.

\section{Study selection}

Studies were selected if they were randomised controlled trials that evaluated the effects of depression screening on diagnosis, treatment, or clinical outcomes in adults in primary care settings.

\section{Data extraction}

Data were extracted on the number of patients, type of intervention, screening instrument, method of administration of the instrument, feedback of screening results to the provider, length of follow up, study quality, and outcomes. Outcomes were diagnosis and treatment rates and rates of persistent depression.

\section{Main results}

14 trials (7739 patients) met the inclusion criteria. 8 trials evaluated screening and feedback of results; 3 trials evaluated screening, feedback, and general or specific treatment advice to providers; and 3 trials evaluated screening, feedback, treatment advice, and assistance to practices to develop systematic means of improving treatment and follow up. Of 7 trials that reported rates of diagnosis, 4 showed greater recognition of depression with screening than with usual care. Of 9 trials that reported rates of treatment, 3 showed greater rates with screening than with usual care. Of 10 trials that reported patient outcomes, 5 showed more improvement in outcomes with screening than with usual care. 7 trials could be combined by meta-analysis. Persistent depression was decreased in patients who received screening. When 1 strongly positive trial causing heterogeneity was removed from the meta-analysis, the reduction in persistent depression remained. Interventions that incorporated systematic means of improving treatment and follow up showed the greatest effect.
For correspondence: Dr M P Pignone,

University of North Carolina at Chape

Hill, Chapel Hill, NC USA. Email pignone@med.unc.edu.

\section{Conclusion}

Screening for depression in adults and feedback to health care providers reduces the risk for persistent depression.

\section{COMMENTARY}

In 1996, the US Preventive Services Task Force (USPSTF) concluded that insufficient evidence existed to recommend routine screening for depression in adult primary care practice. Pignone $e t$ al updated the USPSTF systematic review by incorporating studies to August 2001. They concluded that screening and feedback increase the recognition of depression by a factor of 2 to 3 (absolute increase 10\% to 47\%). Some studies found an increase in the rate of treatment of depression in the intervention group, while others found no difference. Similar variability occurred in the clinical outcomes, notably the prevalence of depression at follow up. When the studies were pooled by meta-analysis, the intervention groups had a $10 \%$ to $13 \%$ reduction in relative risk and a $7 \%$ to $9 \%$ absolute decrease in the prevalence of persistent depression. This led the USPSTF to recommend screening for depression, citing good evidence that screening is beneficial, particularly when combined with effective follow up and treatment.

Why did the USPSTF recommendations change from those of 6 years ago? They did so primarily because of several larger studies released since the previous report, which account for more than half of the "weight" in the weighted meta-analysis.

What should we conclude from these studies? 2 conclusions seem warranted. Firstly, screening for depression with feedback of results is important, because it dramatically increases the recognition of depression. However, studies that relied on feedback, even when combined with treatment advice, generally did not have an effect on clinical outcomes. Therefore, the second conclusion is that the system of care needs to be reorganised ${ }^{1}$ to support the screening and recognition, which can be accomplished through such methods as quality improvement. One approach to support that has worked well is the use of a care manager for depression. ${ }^{2}$ Such system change is not only more likely to be beneficial, it is also more likely to endure over time.

Scott E Sherman, MD, MPH

VA Center for the Study of Healthcare Provider Behavior Sepulveda, California, USA

1 Solberg LI, Korsen N, Oxman TE, et al. The need for a system in the care of depression. I Fam Pract 1999;48:973-9.

2 Simon GE, VonKorff M, Rutter C, et al. Randomised trial of monitoring, feedback, and management of care by telephone to improve treatment of depression in primary care. BMJ 2000;320:550-4. 